\title{
Correction to: Hum Gene Ther 2016;27(1):19-31
}

In the JANUARY 2016 ISSUE of Human Gene Therapy (vol. 27, no. 1, pp. 19-31), the article entitled, "Therapeutic rAAVrh10 Mediated SOD1 Silencing in Adult SOD1 ${ }^{\text {G93A }}$ Mice and Nonhuman Primates," by Dr. Florie Borel et al., contains an error in the Materials and Methods section.

The two copies of miR-SOD1 are actually located in the 3'UTR, and not in the intron, so the sentence that reads "The CB-miR-SOD1 construct contains the same CB-GFP cassette, with the addition of two copies of the artificial miRNA designed to target human SOD1 (miR-SOD1) located within the first intron of the GFP gene." should actually be written as "The CB-miR-SOD1 construct contains the same CB-GFP cassette, with the addition of two copies of the artificial miRNA designed to target human SOD1 (miR-SOD1) located within the 3'UTR of the GFP gene."

The online version of the article has been corrected to reflect this change.

The authors wish to apologize for the error. 